\title{
Communication at the End of Life
}

\author{
Hideki Onishi, M.D., Ph.D. \\ Department of Psycho-oncology, Saitama Medical University International Medical Center, Saitama, Japan
}

End-of-life patients experience physical, mental, social, and existential distress. While medical personnel provide medication and care to alleviate patients' distress, listening to and interacting with patients remains essential for understanding their psychological condition. The most important tool, though difficult to implement in practice, is end-of-life discussion (EOLD). EOLD has been shown to have positive effects on end-of-life treatment choices, achievement of patients' life goals, improvements in the quality of life of patients and their families, and the prevention of depression and complicated grief among bereaved family members. EOLD is not often undertaken in clinical practice, however, due to hesitancy among medical personnel and patients for various reasons. In order to conduct an EOLD, the patient's judgment, psychiatric illnesses such as delirium and depression, and psychological issues such as the side effects of psychotropic drugs, denial, and collusion must be evaluated. Open and honest conversation, treatment goal setting, the doctor's familiarity with the patient's background, and attentiveness when providing information are important elements for any dialogue. Meaning-centered psychotherapy was developed to alleviate the existential distress of cancer patients, and its application may promote EOLD. The future development of meaning-centered psychotherapy in practice and in research is expected to further promote EOLD.

Key Words: Communication, Advance directives, Delirium, Advance care planning, Major depressive disorder, Psychotherapy denial
Correspondence to

Hideki Onishi

ORCID:

https://orcid.org/0000-0003-2949-2734

E-mail: honishi@saitama-med.ac.jp

\section{INTRODUCTION}

The purpose of palliative care is to relieve the various forms of distress experienced by patients suffering from life-threatening illnesses and to improve the quality of life (QOL) of patients and their families. While medical personnel provide medication and care to alleviate distress, it is also essential to listen to patients and engage them in a continuing dialogue. The most important tool, though difficult to apply in practice, is end-of-life discussion (EOLD) [1]. EOLD helps patients come to an understanding of the limits of their lives, set their final life goals, and determine the environment in which they wish to spend their final days and what medical care they wish to receive. Such decisions affect not only the mental state of patients but also that of their family members. EOLD can be very stressful for healthcare professionals and patients, however. Doctors must ask the patients, with whom they have worked together to decide on treatment, about their wishes for their end of life after informing them that the effectiveness of treatment has decreased. In other words, doctors must inform patients about their poor prognosis in order to decide on subsequent treatment options, including palliative care. Patients must also understand their prognosis when making end-oflife decisions. It is not uncommon for both patients and medi- 
cal professionals to be aware of these issues without seriously discussing them due to the magnitude of stress involved in having such discussions.

Herein, the effects of EOLD on end-of-life patients and their families, the factors that hinder EOLD, the basic knowledge required to conduct EOLD, and a checklist of notable points about EOLD are discussed in detail.

\section{WHY IS A DIALOGUE WITH END- OF-LIFE PATIENTS IMPORTANT? (TABLE 1)}

EOLD between end-of-life patients and physicians is essential for improving the QOL of patients and their families. According to a study on the effects of EOLD on the QOL of patients and their families, as well as on the treatment patients receive in the week before their deaths, those who participated in EOLD selected aggressive treatments such as mechanical ventilation, resuscitation, and admission to the intensive care unit (ICU) at a significantly lower rate and were more likely to register for hospice at an early stage. Aggressive treatments were found to reduce the patients' QOL and increase the risk of the family members developing depression after a period of bereavement. In contrast, a longer stay in a hospice corresponded to a higher QOL for patients, which in turn led to improvements in the QOL of patients' families. Furthermore, the incidence of depression or anxiety disorders did not increase in patients who participated in EOLD [2]. That study revealed that physician-patient EOLD has a positive impact on patients' treatment policy decisions, the QOL of patients and families, and the likelihood of bereaved family members developing psychiatric illnesses. In addition, EOLD does not increase patients' risk of developing depression or anxiety.

In another study, patients who prepared advance directives were found to disproportionately prioritize comfort and tended not to want aggressive treatment [3]. It has also been reported that conducting EOLD improved patients' quality of death and end-of-life care and reduced depression and complicated grief among bereaved family members [4].

Cancer patients often show a loss of judgment resulting from a sudden deterioration in their general condition at the end of life. While a study of hospitalized cancer patients showed that many patients still exhibited good judgment at the time of their final admission, $40 \%$ of those patients eventually lost the ability to make decisions and entrusted EOLD to a proxy. These patients often receive aggressive life-sustaining treatments such as mechanical ventilation, artificial nutrition, chemotherapy, and ICU treatment and thus lose the opportunity to improve their end-of-life care. Furthermore, decision-making via a proxy makes dialogue with the physician difficult, resulting in delays related to important medical decisions and stress for the proxy [5].

Research on the content of discussions has found that the following five items are most important when conducting EOLD: priorities in care, values, prognosis, fears and concerns, and questions regarding the goals of care. Patients' satisfaction has been found to increase when comprehensive answers are given to questions about these five items [6]. According to

Table 1. Impact of End-of-Life Discussions on Patients and Their Families.

Impact on patients

Fewer aggressive treatment choices such as mechanical ventilation, resuscitation, and ICU admission

Greater desire to be comfortable

Earlier hospice enrollment

Similar incidence of depression and anxiety disorders to that in the non-EOLD group

(When determined by proxy) Lower rate of choosing aggressive treatment

Impact of family/bereaved

Decreased incidence of depression among bereaved family members

Decreased incidence of complicated grief among bereaved family members

(When determined by proxy) Leads to stress in proxy
Wright 2008 [2], Silveira 2010 [3]

Silveira 2010 [3]

Wright 2008 [2]

Wright 2008 [2]

Zaros 2013 [5]

Yamaguchi 2017 [4]

Yamaguchi 2017 [4]

Zaros 2013 [5]

EOLD: end-of-life discussion. 
a survey of cancer-bereaved family members, the problems experienced by bereaved family members who seek specialized treatment are often a result of regrets about treatment and the lack of sufficient communication with the patient during their lifetime [7].

Some healthcare professionals believe that patients will not be distressed if the truth of their prognosis is withheld from them, which leads to the potential censorship of information in an attempt to protect patients from harmful, sad, or negative news. As a result, honest disclosure is not made during each stage of a patient's illness, from diagnosis to discussion of treatment benefits, recurrence, and end-of-life care. Hiding a patient's actual condition from them causes great confusion among patients, their relatives and friends, as well as other healthcare professionals. It may appear to be beneficial while the patient's general condition is stable; however, failure to convey the correct information deprives the individual patient and their family members of the opportunity to restructure and adapt their daily lives to obtain more achievable goals and develop more realistic hopes and aspirations [8].

\section{FACTORS THAT IMPEDE DIALOGUE WITH END-OF-LIFE PATIENTS}

As mentioned above, physician-patient EOLD is important for making decisions about end-of-life treatment options, improving the QOL of patients and their families, and preventing bereaved family members from developing psychiatric illnesses, such as depression and complicated grief. In reality, however, patients often die without engaging in such dialogue. Accord- ing to a multicenter study from the United States, fewer than $40 \%$ of patients engage in EOLD [2]. Furthermore, a paper on the current state of EOLD among cancer patients initially admitted to palliative care units in East Asia (South Korea, Japan, Taiwan) revealed that the proportion of patients who were told that they were dying was $19.6 \%$ in Korea and only $4.8 \%$ in Japan [9].

Below are the reasons we believe this situation occurs (Table 2).

\section{Factors involving the physician}

Some medical workers believe that they risk depriving patients of their hopes by talking about the end of life. In addition, they often struggle with the timing of when to initiate a discussion about the end of life and wait to receive a signal from the patient or perceive intuitively when the time is appropriate [10].

EOLD may be avoided due to difficulties predicting the course and prognosis of the disease. In non-malignant, lifethreatening illness, in particular, it is difficult to predict when the end of life will occur $[11,12]$.

In addition, medical staff members are often placed in situations where they are required to share a patient's prognosis despite feeling uncertain as to how to go about doing it and that they were not trained to conduct such discussions [13].

\section{Factors involving the patient}

Patients may avoid initiating a dialogue about the end of life if they believe that someone on the medical staff will begin the conversation. However, as mentioned above, medical personnel are also hesitant to initiate EOLD. Thus, both parties may

Table 2. Factors that Hinder End-of-Life Discussions.

\begin{tabular}{ll}
\hline \multicolumn{1}{c}{ Factors } & \multicolumn{1}{c}{ References } \\
\hline Medical staff-related factors & Almack 2012 [10] \\
Feelings of depriving the patient of hope & Almack 2012 [10], Clayton 2005 [14] \\
Thinking the patient will initiate the conversation & Hancock [11] \\
Difficulty predicting the disease course and prognosis & Christakis 1998 [13] \\
Inadequate training for communicating the prognosis & \\
Patient-related factors & Almack 2012 [10], Clayton 2005 [14] \\
Thinking that medical personnel will initiate the conversation & Zaros 2013 [5] \\
Deterioration in general condition & Stiefel 2017 [15] \\
Medical staff- and patient-related factors &
\end{tabular}


lose the opportunity to talk to each other due to the belief that the other party will initiate the conversation at some point [10].

In a study of patients, caregivers, and healthcare professionals on the who and when of EOLD initiation, the largest share of patients preferred option 2 ("healthcare professionals provide opportunities to talk to patients about the future") among the following four options: 1) the patient/carer raises issues, 2) healthcare professionals provide opportunities to talk to patients about the future, 3) healthcare professionals discuss what they want to know about patients/carers, and 4) healthcare professionals start a dialogue when they feel the patient/ carer is ready. Many patients and families want healthcare professionals to initiate EOLD [14]. However, since doctors often prefer for patients to initiate the dialogue, there is a high likelihood of misunderstandings that result in EOLD never taking place.

As mentioned above, cancer patients often show a loss of judgment as a result of a sudden deterioration in their general condition at the end of life. According to a study of inpatients with cancer, $40 \%$ of patients eventually lost the ability to make judgments and had to entrust EOLD to a proxy [5].

\section{Factors related to both medical personnel and patients}

The term "collusion" refers to a situation in which medical personnel and patients unknowingly collaborate. Collusion is an unconscious bond in which both parties unknowingly participate in a conspiracy to avoid addressing underlying psychological factors.

Collusion is most likely to occur when the parties involved have irresolvable issues and in various clinical settings, but it has been recognized that collusion is also likely to occur in end-of-life care [15]. An example of this situation is when a patient with separation anxiety faces the prospect of death and requests assisted suicide. This request can be understood as an attempt to reduce the patient's feeling of psychological tension associated with death. However, if the patient were to be treated by a healthcare professional who themselves experiences separation anxiety, then the staff member is likely to experience stress as a result of the patient's request and reject it. Thus, the psychological problem underlying the request remains unexplored by both parties [15].

\section{WHEN IS THE BEST TIME TO COMMENCE EOLD?}

EOLD should be commenced at the appropriate time, and qualitative research suggests that it should be initiated early and routinely. However, in the early stages of a patient's illness, the patient and his or her family may not be able to accept the information provided as they focus on fighting the disease [16].

In addition, at the end of life, some patients develop delirium and show a loss of judgment. According to a study, about $70 \%$ of patients for whom decision-making was required had lost their ability to make decisions [3]. In addition, one study found that about $40 \%$ of patients experienced a loss of judgment in the course of their hospitalization [5]. Thus, commencing EOLD early is preferable.

Cancer patients often experience a sudden deterioration in their general condition as the disease progresses in the latter half of the terminal stage, and it can be especially difficult to identify the terminal stage in heart disease patients [12]. This also indicates that medical personnel should initiate EOLD as early as possible to ensure that these important discussions are conducted.

\section{A CHECKLIST FOR EOLD}

\section{Judgment (Table 3 )}

Engagement in an EOLD is conditional on the patient showing appropriate judgment. However, it is difficult to determine if clinicians have fully examined a patient's ability to make judgments. It has been shown that healthcare professionals in a hospital setting find patients to be incapable of making judgments in as few as $25 \%$ of cases [17]. Therefore, healthcare professionals should always consider whether the patient has the necessary judgment to commence EOLD.

To ensure that a patient is able to make judgments, the physician should examine whether the patient is capable of the following: (1) communicating a choice, (2) understanding the relevant information, (3) comprehending the situation and its consequences, and (4) making a rational decision about treatment options [18]. 
Table 3. A Checklist for Initiating an End-of-Life Discussion.

\begin{tabular}{ll}
\hline \multicolumn{1}{c}{ Checklist } & \multicolumn{1}{c}{ References } \\
\hline Necessary point & \\
Absence of judgment & Appelbaum 2007 [18] \\
Psychiatric illness & \\
Delirium & Onishi in press [19] \\
Depression & Onishi in press [19] \\
Medication & \\
Adverse effects of psychotropic drugs & Ishida 2020 [20] \\
(akathisia, etc.) & \\
Psychological issues & \\
Denial & Hughes 1986 [21] \\
Collusion & Stiefel 2017 [15] \\
\hline
\end{tabular}

\section{Things other than judgment to be checked (Table 3)}

\section{1) Delirium}

The presence or absence of delirium should always be confirmed. Delirium is an acute brain dysfunction that often goes overlooked, particularly in cases of hypoactive delirium. The presence of delirium significantly lowers the QOL of patients and affects their judgment, but there are many cases in which delirium can be treated by identifying the cause and providing an appropriate intervention. In particular, patients suffering from hypoactive delirium may appear quiet at first glance. However, if hypoactive delirium in a patient is overlooked, the patient is likely to exhibit a low level of consciousness and attention during an EOLD, which may obscure the patient' $s$ wishes and goals. In addition, there has been at least one case in which a patient thought to have terminal delirium was found to have Wernicke's encephalopathy instead, and thiamine administration improved the patient's cognitive ability [19].

\section{2) Depression}

After confirming that a patient does not have delirium, he or she should be examined for depression. Depression is a difficult condition for patients and carries an association with changes in treatment policy and an increase in the suicide rate. Drug therapy is likely to improve the symptoms of depression. Notably, there are many cases in which patients referred for an examination for depression also suffer from delirium [19]. As such, physicians should be sure to check for delirium even after depression is diagnosed.

\section{3) Adverse effects of psychotropic drugs}

At the end of life, psychotropic drugs are often prescribed to treat nausea and impaired consciousness. Some of these drugs may cause distressing side effects such as akathisia. Symptoms such as pain when sitting and wandering are often experienced by patients with akathisia, and even though many patients are unable to get up at the end of life, akathisia still occurs in such conditions [20]. Akathisia in a patient who is bedridden for most of the day can cause significant distress since the $\mathrm{pa}^{-}$ tient is almost unable to move. It may be difficult for medical personnel to identify akathisia based on its symptoms when the patient's movements are already so restricted. Thus, the potential for patients who are administered psychotropic drugs to develop akathisia should be kept in mind. Akathisia can also occur in patients after leg amputation [20], as found in another study in which a leg amputation patient experienced suicidal ideation due to the suffering involved and asked, "Can I die now?" Such sentiments would have a significant impact on EOLD. Therefore, since the symptoms of akathisia are extremely painful and affect a patient's mental state if left untreated, failure to address the symptoms of akathisia immediately can affect EOLD.

\section{4) Denial}

Denial is a defense mechanism that works to protect the ego in which one acts as if he or she faces no threat, but it is also said to be an adaptive strategy to protect oneself from events and emotions caused by suffering [21]. Clinically, patients in denial do not ask questions about their diagnosis or future treatment and act as if they faced no serious health problems [21]. In a survey of lung cancer patients, denial was observed in more than $80 \%$ of patients, of whom $3 \%$ were in strong denial, indicating that denial is a common phenomenon [22].

In situations in which patients clearly deny their current condition, denial should be assumed and measures such as the postponement of EOLD should be taken.

\section{5) Collusion}

Patients and healthcare professionals unknowingly collaborate to avoid directly addressing the psychological underpin- 
nings of an apparent problem. Supervision has been found to be effective at solving this problem, as exemplified in a previous study [15].

\section{6) Physical and psychological state of patients' family members}

In addition, it is necessary to understand the physical and psychological state of patients' family members, as they are often regarded as care providers alongside medical personnel. Once someone is diagnosed with a life-threatening illness, various physical and psychological burdens are placed on the family members of the patient. In other words, family members become "second-order patients" who are also the subject of treatment and care. Poor psychological health among family members can thus affect dialogues with healthcare professionals. Furthermore, it is not uncommon for family members to suffer from depression [23]. In addition, there have been cases in which family members of patients develop cancer and an asymptomatic thiamine deficiency. It is also not uncommon for families to experience financial problems related to their family member's illness. Professional approaches have been devised for addressing the above problems, and the psychological states of terminal patients' family members should be monitored as such [7].

\section{WHAT MEDICAL PERSONNEL SHOULD BE AWARE OF WHEN INTERACTING WITH END-OF-LIFE PATIENTS}

\section{Differences in understanding between medical personnel and patients}

There is a considerable difference between the understanding of medical conditions by medical personnel and by patients and their families. Physicians should make sure not to overestimate patients' and families' understanding of a medical condition and instead ensure that they understand the progress of the illness up to that point in time, the status of the prognosis, and what is expected to change in the future [24].

\section{Correcting the beliefs of medical staff}

1) Patients will initiate the EOLD

Healthcare professionals who are concerned about the timing of when to initiate an EOLD may believe that the patient will initiate the dialogue [10]. However, the patient typically expects a medical staff member to initiate the dialogue, which can result in missing the appropriate window within which to conduct an EOLD. As mentioned above, medical personnel should keep in mind that initiating an EOLD will benefit the patient and adjust their expectations if they believe that patients will start such discussions.

\section{2) EOLD may harm the patient}

Some healthcare professionals believe that they risk crushing the hopes of terminal patients by discussing where and how to receive end-of-life care [10]. According to a study that examined whether interviews with patients and care providers about death, dying, and bereavement caused pain or harm, fewer than $2 \%$ of end-of-life patients felt a great deal of stress as a result of the interviews and only $7.1 \%$ experienced some stress. Based on these results, medical personnel should view EOLD as positive given that EOLD-related stress is typically minimal [25].

\section{3) EOLD may lead to the onset of mental illness}

There is a concern among some healthcare professionals that EOLD may lead to feelings of desperation in the patient and that a patient's family may also ask medical personnel to stop talking about the patient's prognosis for the same reason.

Studies on EOLD and the onset of mental illness have shown no significant differences in the incidence of mental illness according to the absence or presence of EOLD [2]. EOLD can also reduce the rate at which bereaved family members develop depression and complicated grief [4]. 


\section{WHAT DOCTORS CONSIDER NECESSARY TO FACILITATE DIALOGUE WITH END-OF-LIFE PATIENTS}

Qualitative research on the end of life has revealed that medical personnel should consider the following strategies when conducting EOLD: open and honest conversation, setting treatment goals, and balancing hope with reality [16].

\section{WHAT A PATIENT WANTS IN EOLD WITHA PHYSICIAN}

When conducting an EOLD, the patient wants to be treated as an individual, to be listened to, to build relationships with medical personnel, and to be provided with holistic care in a privacy-friendly environment by sympathetic medical professionals. The contents of EOLD desired by the patient include sympathy from medical personnel; familiarity with the background of the patient by the physician; the patient's readiness status being considered when providing information; initiation of the conversation by a medical professional; and honesty, intelligibility, and attentiveness when providing information [26].

\section{MEANING-CENTERED PSYCHOTHERAPY FOR ADVANCED CANCER PATIENTS}

Spiritual well-being and fostering a sense of meaning are important for patients with advanced cancer [27], and interventions should be developed that address these factors. Meaning-centered psychotherapy, with a particular focus on the needs of patients with advanced cancer, was developed to meet these needs.

This treatment method was developed based on Frankl's logotherapy and meaning-based psychotherapy. Viktor Frankl, an Austrian psychiatrist, was a Holocaust survivor interned by the Nazis in concentration camps, including Auschwitz, during World War II. His main work was Man's Search For Meaning [28]. Frankl's basic concepts included the following three elements: (1) meaning of life, in that life has meaning; (2) will to meaning, in that humans have a desire for meaning and meaning is a basic motivation of human behavior; and (3) freedom of will, in that one is free to seek meaning. In other words, an individual has the freedom to determine his or her attitude toward suffering. The three sources of meaning are creativity, experience, and attitude. Frankl gives an example from a busy advertising designer in his book, whose attitude was "say yes to life in spite of everything" [29]. The designer was paralyzed due to a spinal cord tumor, which interrupted his professional life and left him unable to live an active lifestyle. He understood the value of his experiences by reading in the hospital and having lively conversations with other patients. When his condition progressed and he could not continue such activities due to his reduced life expectancy, he tried to lower the burden on Dr. Frankl by limiting his intake of medication as much as possible. In the case of this patient, Frankl reports an example of compassion that was still evident just hours before the patient's death, thus demonstrating a positive attitude toward the end of life.

Frankl's concept has positive implications for cancer patients who face issues related to persistence, hope, understanding of their condition, and imminent death.

Meaning-centered therapy consists of a 7-week intervention program, provided in a manual form, for patients with advanced cancer and aims to help patients find meaning, peace, and purpose in life when faced with its limits as their illnesses progress. This treatment has also been demonstrated by a randomized controlled trial to be beneficial for spiritual suffering and QOL in patients with advanced cancer [30].

Our hospital also provides group psychotherapy based on the essential elements of this method. By undertaking group psychotherapy with a psychiatrist as a facilitator and a psychooncologist in attendance, some patients who are desperate or unable to face their illness confess to feeling as though they are "being tested by life," while others experience changes in disposition that indicate post-traumatic growth through comments such as "I became kind to people," "As a result of earnest discussions at this meeting, I was able to understand the depth of my life," and "I will die before the age of 60 , and while I may have been unlucky, my life is not unfortunate." 


\section{CONCLUSION}

As supported by extensive research, EOLD between medical personnel and patients enhances the QOL of end-of-life patients, improves the quality of care experienced by patients' families, and helps to prevent psychiatric illness in bereaved family members. However, due to various factors, EOLD remains insufficient in many contexts. We hope that reading this review will help healthcare professionals overcome barriers to conducting EOLD, understand what EOLD requires, and improve communication with patients.

As a clinician, I have been working with cancer patients for 35 years. Even when patients find themselves in a difficult situation, I believe it is possible for them to adapt to it, develop the ability to find higher purpose in life, and grow personally.

\section{ACKNOWLEDGMENTS}

I would like to express my sincere gratitude to Dr. Jae-Hon Lee and Dr. Mayumi Ishida for their generous support in preparing this review.

\section{CONFLICT OF INTEREST}

No potential conflict of interest relevant to this article was reported.

\section{REFERENCES}

1. Brighton LJ, Bristowe K. Communication in palliative care: talking about the end of life, before the end of life. Postgrad Med J 2016;92:46670.

2. Wright AA, Zhang B, Ray A, Mack JW, Trice E, Balboni T, et al. Associations between end-of-life discussions, patient mental health, medical care near death, and caregiver bereavement adjustment. JAMA 2008;300:1665-73.

3. Silveira MJ, Kim SY, Langa KM. Advance directives and outcomes of surrogate decision making before death. N Engl J Med 2010;362:1211-8.

4. Yamaguchi T, Maeda I, Hatano Y, Mori M, Shima Y, Tsuneto S, et al. Effects of end-of-life discussions on the mental health of bereaved family members and quality of patient death and care. J Pain Symptom Manage 2017;54:17-26.e1.

5. Zaros MC, Curtis JR, Silveira MJ, Elmore JG. Opportunity lost: end-of-life discussions in cancer patients who die in the hospital. J Hosp Med 2013;8:334-40.

6. You JJ, Dodek P, Lamontagne F, Downar J, Sinuff T, Jiang X, et al. What really matters in end-of-life discussions? Perspectives of patients in hospital with serious illness and their families. CMAJ 2014;186:E679-87.

7. Ishida M, Onishi H. How can documentation of caregivers offer more than one-way care by healthcare professionals? J Clin Oncol 2021:JCO2101382.

8. Fallowfield LJ, Jenkins VA, Beveridge HA. Truth may hurt but deceit hurts more: communication in palliative care. Palliat Med 2002;16:297303.

9. Yamaguchi T, Maeda I, Hatano Y, Suh SY, Cheng SY, Kim SH, et al. Communication and behavior of palliative care physicians of patients with cancer near end of life in three east Asian countries. J Pain Symptom Manage 2021;61:315-322.e1.

10. Almack K, Cox K, Moghaddam N, Pollock K, Seymour J. After you: conversations between patients and healthcare professionals in planning for end of life care. BMC Palliat Care 2012 17;11:15.

11. Hancock K, Clayton JM, Parker SM, Wal der S, Butow PN, Carrick S, et al. Truth-telling in discussing prognosis in advanced life-limiting illnesses: a systematic review. Palliat Med 2007;21:507-17.

12. Lynn J. Perspectives on care at the close of life. Serving patients who may die soon and their families: the role of hospice and other services. JAMA 2001;285:925-32.

13. Christakis NA, Iwashyna TJ. Attitude and self-reported practice regarding prognostication in a national sample of internists. Arch Intern Med 1998;158:2389-95.

14. Clayton JM, Butow PN, Tattersall MH. When and how to initiate discussion about prognosis and end-of-life issues with terminally ill patients. J Pain Symptom Manage 2005;30:132-44.

15. Stiefel F, Nakamura K, Terui T, Ishitani K. Collusions between patients and clinicians in end-of-life care: Why clarity matters. J Pain Symptom Manage 2017;53:776-82.

16. Granek L, Krzyzanowska MK, Tozer R, Mazzotta P. Oncologists' strategies and barriers to effective communication about the end of life. J Oncol Pract 2013;9:e129-35.

17. Raymont V, Bingley W, Buchanan A, David AS, Hayward P, Wessely S, et al. Prevalence of mental incapacity in medical inpatients and associated risk factors: cross-sectional study. Lancet 2004;364:1421-7. 
18. Appelbaum PS. Clinical practice. Assessment of patients' competence to consent to treatment. N Engl J Med 2007;357:1834-40.

19. Onishi H, Sato I, Uchida N, Takahashi T, Furuya D, Ebihara Y, et al. High proportion of thiamine deficiency in referred cancer patients with delirium: a retrospective descriptive study. Eur J Clin Nutr. In press 2021.

20. Ishida M, Imanishi J, Yazawa Y, Sunakawa Y, Torigoe T, Onishi H. "Phantom akathisia" in an amputated leg of a sarcoma patient: a case report. Biopsychosoc Med 2020;14:4.

21. Hughes J. Denaial in cancer patients. In: Stoll BA, ed. Coping with cancer stress. Dordrecht; Boston: Nijhoff; Hingham, MA, USA: Distributors for the U.S. and Canada, Kluwer Academic Publishers;1986. p. 63-9.

22. Vos MS, Putter H, van Houwelingen HC, de Haes HC. Denial in lung cancer patients: a longitudinal study. Psychooncology 2008;17:1163-71.

23. Braun M, Mikulincer M, Rydall A, Walsh A, Rodin G. Hidden morbidity in cancer: spouse caregivers. J Clin Oncol 2007;25:4829-34.

24. Hancock K, Clayton JM, Parker SM, Walder S, Butow PN, Carrick S, et al. Discrepant perceptions about end-of-life communication: a systematic review. J Pain Symptom Manage 2007;34:190-200.

25. Emanuel EJ, Fairclough DL, Wolfe P, Emanuel LL. Talking with terminally ill patients and their caregivers about death, dying, and bereavement: is it stressful? Is it helpful? Arch Intern Med 2004;164:1999-2004.

26. Abdul-Razzak A, You J, Sherifali D, Simon J, Brazil K. 'Conditional candour' and 'knowing me': an interpretive description study on patient preferences for physician behaviours during end-of-life communication. BMJ Open 2014;4:e005653.

27. Breitbart W. Spirituality and meaning in supportive care: spirituality- and meaning-centered group psychotherapy interventions in advanced cancer. Support Care Cancer 2002;10:272-80.

28. FrankI VE. Man's search for meaning. London:Rider;2020.

29. FrankI VE. Yes to life: in spite of everything. London:Rider;2020.

30. Breitbart W, Poppito S, Rosenfeld B, Vickers AJ, Li Y, Abbey J, et al. Pilot Randomized Controlled Trial of Individual Meaning-Centered Psychotherapy for Patients With Advanced Cancer. J Clin Oncol 2012;30:1304-9. 